\title{
Aggressive Aortopathy in neonatal Marfan syndrome
}

\author{
Laura D'Addese $^{1 *}$ D, Rukmini Komarlu² and Kenneth Zahka ${ }^{2}$
}

\begin{abstract}
Background: Neonatal Marfan syndrome is a rare, severe form of Marfan syndrome with a poor prognosis. Surgical intervention to address massive aortic root dilatation is uncommon as dissection rarely occurs, and death invariably results from congestive heart failure and recurrent respiratory infections.

Case presentation: An 11 month old female with a prenatal diagnosis of aortic root dilatation was aggressively treated both medically and surgically. Genetic testing revealed a partial deletion in FBN1 exons 46-50 and an addition 17q11 microdeletion. Valve-sparing aortic root remodeling was successful at eliminating her risk of a type A aortic dissection. Prior to surgery, however, her root caused significant left atrial and left lower pulmonary vein compression, which has not completely resolved.
\end{abstract}

Conclusions: Aortic dilatation occurs rapidly despite aggressive medical management. Root remodeling and atrioventricular valve repair are possible, but the durability of the latter is uncertain. It is possible that our patient's combined gene mutations are exacerbating her disease.

Keywords: Neonatal Marfan syndrome, FBN1 mutation, Aortic dilatation, Aortic dissection

\section{Background}

Neonatal Marfan syndrome (NMFS) is a rare, severe form of Marfan syndrome (MFS) with a poor prognosis and several distinguishing features. Unlike traditional Marfan syndrome, family history is negative in $70-100 \%$ of cases, suggesting a high degree of de novo mutations [1]. Aortic dilatation (AD) occurs in over $80 \%$ of patients, but dissection is quite rare, and death typically occurs within the first year of life from congestive heart failure (CHF) secondary to severe atrioventricular valve (AV) regurgitation and ventricular dysfunction [2]. Systemic findings like diaphragmatic eventration, joint contractures, and severe scoliosis are also more prevalent and may portend a worse prognosis when associated with certain exon mutations [3-5]. Treatment focuses on medical management of heart failure symptoms and surgical interventions are rarely performed given the low risk of $\mathrm{AD}$.

\footnotetext{
* Correspondence: laura.d'addese@gosh.nhs.uk

${ }^{1}$ Cardiology Department, Great Ormond Street Hospital, Great Ormond

Street, London WC1N 3HZ, UK

Full list of author information is available at the end of the article
}

\section{Case presentation}

11 month old female with a prenatal diagnosis of aortic root dilatation. Her mother has ectopia lentis and aortic root dilatation as diagnostic criteria for MFS, but aortopathy panel and chromosome microarray were negative. The initial post-natal echo showed myxomatous, severely prolapsing $\mathrm{AV}$ valves, mild tricuspid regurgitation (TR), mild mitral regurgitation (MR), normal biventricular size and function, and moderate aortic root dilation $(1.46 \mathrm{~cm}$, Z-score 3.90$)$ (Fig. 1). All Z scores were calculated from the Boston Children's Hospital dataset to ensure consistency over time. An aberrant right subclavian artery with Kommerell's diverticulum was also noted. Physical exam was remarkable for marked arachnodactyly, scoliosis, malar hypoplasia, flat corneas, and pectus deformity. She had persistent arterial desaturation due to right diaphragm eventration causing hepatic compression of the right atrium and streaming of inferior vena cava blood across a patent foramen ovale (Fig. 2). Whole exome sequencing demonstrated a partial FBN1 deletion involving exons 46-50 and chromosome microarray showed an additional 17q22 microdeletion. Paternal genetic testing and in silico testing has not be performed.

(C) The Author(s). 2019 Open Access This article is distributed under the terms of the Creative Commons Attribution 4.0 International License (http://creativecommons.org/licenses/by/4.0/), which permits unrestricted use, distribution, and 

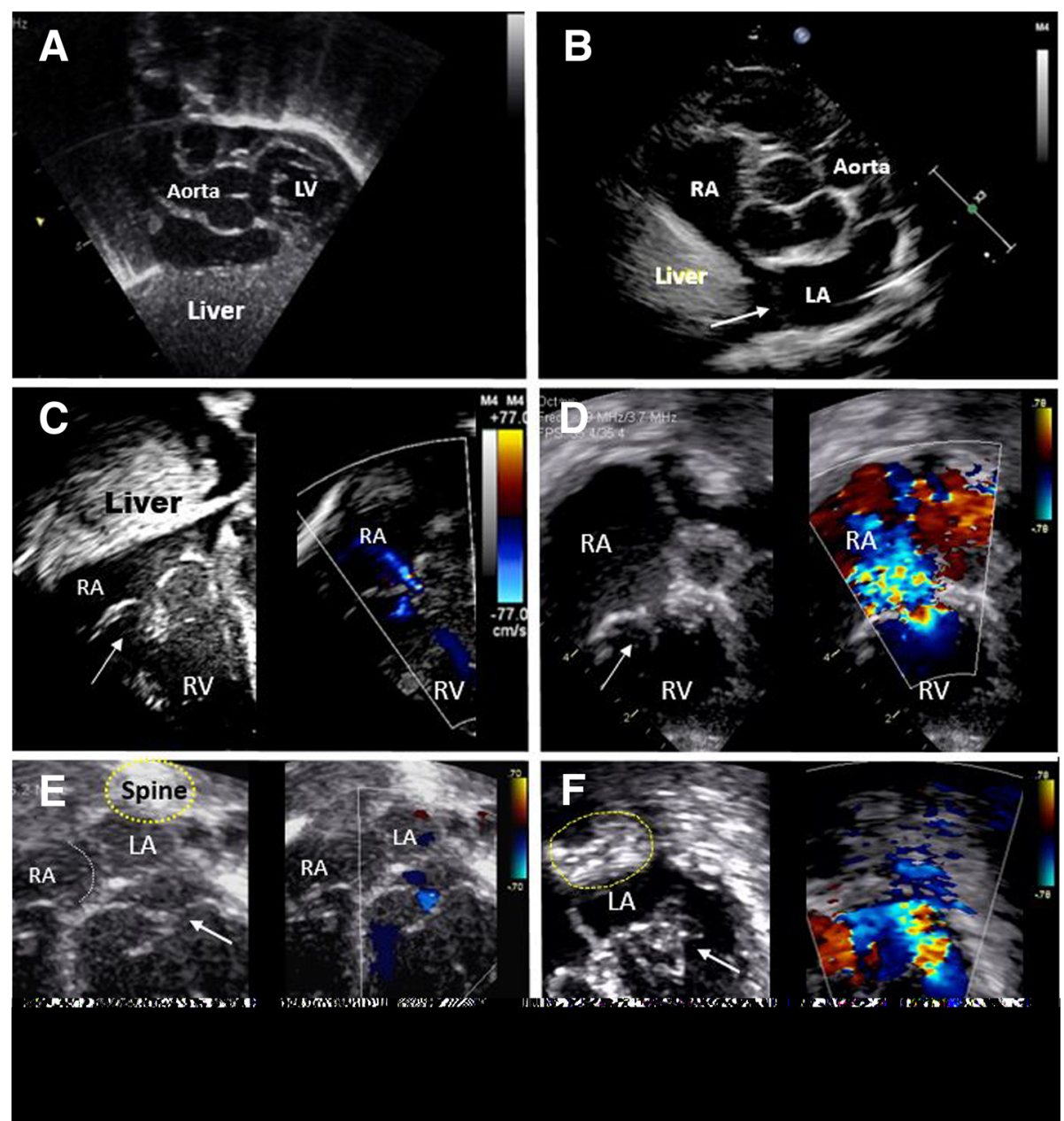

Fig. 1 Transthoracic echocardiogram. a 2D subcostal long axis view: Moderately dilated aortic root (1.46 cm, Z score 3.90). (LV: left ventricle). b 2D parasternal short axis view: Diaphragmatic eventration causing liver protrusion into thoracic the cavity and compression of the right atrium (RA). (LA: left atrium; RVOT: right ventricular outflow tract). c Magnified color compare 4 chamber apical view: Myoxomatous, severely prolapsing tricuspid valve (arrow) with mild tricuspid regurgitation. Liver is visibly compressing the right atrium. (RV: right ventricle). $\mathbf{d}$ Color compare 4 chamber apical view: Myoxomatous, severely prolapsing tricuspid valve with central coaptation gap (arrow) and severe tricuspid regurgitation. e Magnified color compare 4 chamber apical view: Myoxomatous, severely prolapsing mitral valve (arrow) with mild regurgitation. Note the spine compressing the left atrium and the leftward bowing of the atrial septum (dotted line). $\mathbf{f}$ Magnified color compare 4 chamber apical view: Myoxomatous severely prolapsing mitral valve (arrow) with severe regurgitation through a posterior jet

Atenolol (initially $3 \mathrm{mg}$ daily; $\sim 1 \mathrm{mg} / \mathrm{kg}$ ) and losartan (initially $10 \mathrm{mg}$ daily; $\sim 3.2 \mathrm{mg} / \mathrm{kg}$ ) were started at 6 weeks of age following significant progression of her aortic root dimension $(1.93 \mathrm{~cm}$, Z-score +6.96$)$ (Fig. 2). With close heart rate and blood pressure monitoring, the patient tolerated a maximal dose of atenolol $12 \mathrm{mg}$ daily and losartan $25 \mathrm{mg}$ daily. Her AV valve regurgitation remained mild. At 4.5 months of age she was admitted in CHF refractory to maximal medical therapy. She had severe TR, moderate MR, and trivial aortic regurgitation (Fig. 1). Her aortic root measured $2.46 \mathrm{~cm}$ (Zscore of +9.14$)$. She underwent mitral and tricuspid valvuloplasty and right hemidiaphragm plication. Postoperative TEE demonstrated persistent moderate MR.
Serial echocardiograms showed worsening MR and progressive aortic root dilatation. She was re-admitted in $\mathrm{CHF}$ at 9.5 months and underwent a second mitral valvuloplasty with leaflet resection and artificial chordae creation. Her aortic root measured $3.1 \mathrm{~cm}$ (Z-score +13 ). Losartan was switched to irbesartan (initiated at $10 \mathrm{mg}$ to a maximal dose of $40 \mathrm{mg}$ daily) in an effort to attenuate further aortic root growth, but her most recent echo showed further dilation to $3.35 \mathrm{~cm}$ (Z-score + 14.4) and compression of the left atrium and left-sided pulmonary veins between the dilated aortic root and scoliotic spine. This was confirmed by chest CT which revealed near complete obliteration of the left atrium (Fig. 3). Valvesparing aortic root replacement using the Yacoub 


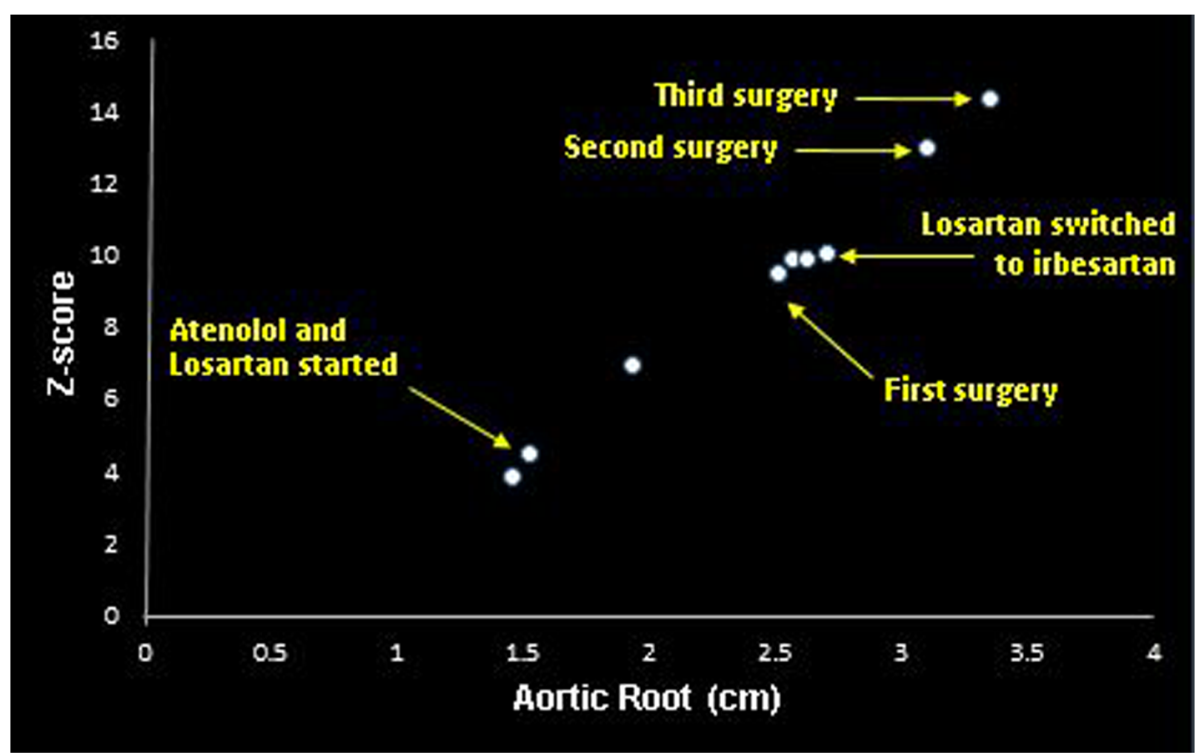

Fig. 2 Aortic root dimensions over time with corresponding medical and surgical interventions

technique was employed with normalization of her aortic root dimension and alleviation of her left atrial compression. Unfortunately, her left lower pulmonary vein stenosis progressed to obliteration of the vein with formation of collateral drainage. The left upper pulmonary vein also became narrowed. Repeat echocardiogram showed normal pulmonary artery pressures and right ventricular function.

\section{Discussion}

Neonatal Marfan syndrome is a rare disorder with a poor prognosis. Aortic root dilation is quite common, but dissection is not and death typically results from $\mathrm{CHF}$ due to severe AV valve regurgitation and ventricular dysfunction [2]. Systemic findings are also more pronounced and occur in a larger proportion of individuals relative to classical MFS. Stheneur et al demonstrated that mutations in exons 25-26, in conjunction with valvular regurgitation and diaphragmatic hernia, are associated with a worse prognosis [5]. Our patient had both these systemic findings, but her partial FBN1 gene mutation involved exons 46-50, which presumably cause less aggressive disease.

Genetic testing also revealed a 17q22 microdeletion. This region contains the NOG gene whose protein product, Noggin, plays a role in proper bone and joint development. Laurell et al described the molecular and clinical phenotype of deletions in this region in the largest case series to date [6]. All six patients had NOG-related skeletal and joint features and a constellation of facial dysmorphologies, while other had conductive hearing loss, intellectual disability, and attention deficient hyperactivity disorder. One patient had aortic arch hypoplasia and deletion of OR4D1, OR4D2, DYNLL2, MKS1, AND MSX2P1, but
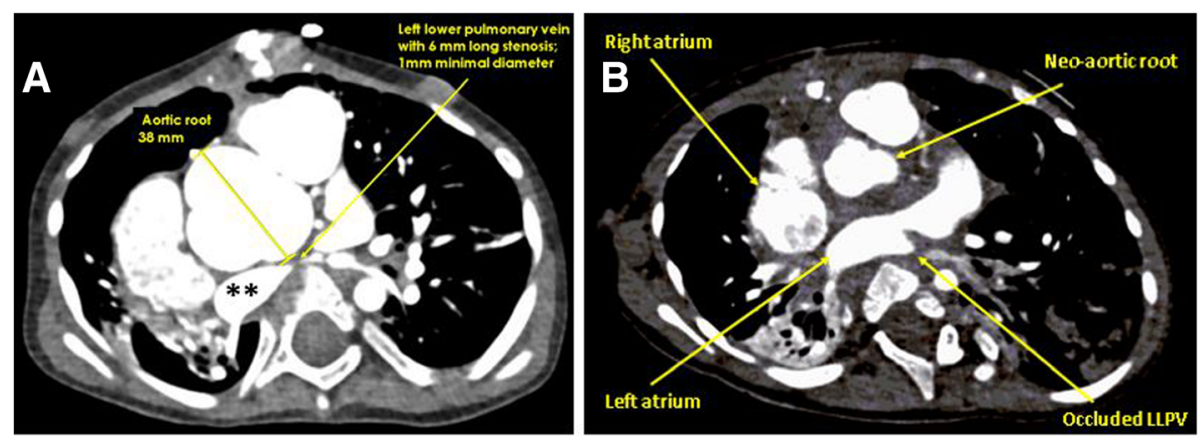

Fig. 3 Chest CTA with contrast. a Pre-operative axial image: Severe aortic root dilatation to almost $4 \mathrm{~cm}$, compression of the left atrium (LA) between the aortic root and spine, and severe left lower pulmonary vein (star) stenosis. (DAO: descending abdominal aorta; RVOT: right ventricular outflow tract). b Post-operative axial imaging: Reduced aortic root dimension, resolution of left atrial compression, and occluded left lower pulmonary vein (arrow). Note the massively dilated right atrium (RA) 
another patient with these same deletions had no cardiac anomalies.

Our patient had rapid aortic root dilatation despite aggressive medical management with beta blocker and angiotensin receptor blocker. She underwent successful valve-sparing root remodeling and AV valvuloplasty, but the durability of the latter is unclear given the highly dysplastic nature of these valves. While she is no longer at risk of type A dissection, she remains at risk for thoracic aortic dilatation and dissection, including her Kommerell's diverticulum. Her left-sided pulmonary vein stenosis is being monitored closely and she does not currently show signs of elevated pulmonary artery pressures or decreased right ventricular function.

\section{Conclusion}

Aortic dilatation in NMFS occurs rapidly despite aggressive medical management. Root remodeling and atrioventricular valve repair are possible, but the durability of the latter is unknown. By repairing her AV valve regurgitation and addressing her aortic root dilatation, we have markedly altered the course of her disease progression. Deletion of specific FBN1 exons is known to carry a worse prognosis, but our patient's deletion is outside this region. Her 17q22 microdeletion also does not account for the aggressive nature of her aortopathy, although it may account for the severity of her skeletal findings. Additional genetic testing is needed to identify any possible synergistic effects on her cardiac phenotype.

\section{Abbreviations \\ AD: Aortic dilatation; AV: Atrioventricular; CHF: Congestive heart failure; MFS: Marfan syndrome; MR: Mitral regurgitation; NMFS: Neonatal Marfan syndrome; TR: Tricuspid regurgitation}

\section{Acknowledgements}

Not applicable.

\section{Author's contributions}

LD drafted the manuscript. RK assisted in reviewing the images for publication and assisted in editing the manuscript. KZ assisted in editing the manuscript. All authors read and approved the final manuscript.

\section{Funding}

No funding was involved in producing this case report.

\author{
Availability of data and materials \\ Data sharing not applicable to this article as no datasets were generated or \\ analyzed during the current study.
}

Ethics approval and consent to participate

Not applicable.

\section{Consent for publication}

The patient's mother provided verbal and written consent for this publication.

\section{Competing interests}

The authors declare that they have no competing interests.

\section{Author details}

${ }^{1}$ Cardiology Department, Great Ormond Street Hospital, Great Ormond Street, London WC1N 3HZ, UK. ²Department of Pediatric Cardiology,

Cleveland Clinic Foundation, 9500 Euclid Avenue, Cleveland, OH 44195, USA.

Received: 18 February 2019 Accepted: 22 May 2019

Published online: 06 June 2019

\section{References}

1. Morse RP, Rockenmacher S, Pyeritz RE, et al. Diagnosis and management of infantile Marfan syndrome. Pediatrics. 1990;86(6):888-95.

2. Shih H, Liu W, Chen T. Neonatal Marfan syndrome - a case report. Acta Cardiol Sin. 2004;20:171-5

3. Ghandi Y, Zanjani KS, Mazhari-Mousavi SE, et al. Neonatal Marfan syndrome: report of two cases. Iran J Pediatr. 2013;23(1):113-7.

4. Phornphutkul C, Rosenthal A, Nadas A. Cardiac manifestations of Marfan syndrome in infancy and childhood. Circulation. 1973;47:587-96.

5. Stheneur C, Faivre L, Collod-Beroud G, et al. Prognosis factors in probands with an FBN1 mutation diagnosed before the age of 1 year. Ped Res. 2011; 69(3):265-70.

6. Laurell T, Lundin J, Anderlid BM, et al. Molecular and clinical delineation of the 17q22 micro deletion phenotype. Euro J Human Gen. 2013;21:1085-92.

\section{Publisher's Note}

Springer Nature remains neutral with regard to jurisdictional claims in published maps and institutional affiliations.
Ready to submit your research? Choose BMC and benefit from:

- fast, convenient online submission

- thorough peer review by experienced researchers in your field

- rapid publication on acceptance

- support for research data, including large and complex data types

- gold Open Access which fosters wider collaboration and increased citations

- maximum visibility for your research: over $100 \mathrm{M}$ website views per year

At BMC, research is always in progress.

Learn more biomedcentral.com/submissions 